\title{
Concerning the instantaneous mass and the extent of an expanding universe
}

\author{
H.J. Fahr ${ }^{1, \star}$ and M. Heyl ${ }^{2}$ \\ ${ }^{1}$ Institute for Astrophysics and Extraterrestrial Research (now part of Argelander-Institut für Astronomie), University of \\ Bonn, Auf dem Hügel 71, 53121 Bonn, Germany \\ 2 Deutsches Zentrum für Luft und Raumfahrt (DLR), Königswinterer Straße 522, 53227 Bonn, Germany
}

Received 2005 Oct 6

Key words cosmology - mass of universe; cosmology - cosmic density

\begin{abstract}
In this article we want to answer the cosmologically relevant question what, with some good semantic and physical reason, could be called the mass $M_{\mathrm{u}}$ of an infinitely extended, homogeneously matter-filled and expanding universe. To answer this question we produce a space-like sum of instantaneous cosmic energy depositions surrounding equally each spacepoint in the homogeneous universe. We calculate the added-up instantaneous cosmic energy per volume around an arbitrary space point in the expanding universe. To carry out this sum we use as basic metrics an analogy to the inner Schwarzschild metric applied to stars, but this time applied to the spacepoint-related universe. It is then shown that this leads to the added-up proper energy within a sphere of a finite outer critical radius defining the point-related infinity. As a surprise this radius turns out to be reciprocal to the square root of the prevailing average cosmic energy density. The equivalent mass of the universe can then also be calculated and, by the expression which is obtained here, shows a scaling with this critical radius of this universe, a virtue of the universe which was already often called for in earlier works by E.Mach, H.Thirring and F.Hoyle and others. This radius on the other hand can be shown to be nearly equal to the Schwarzschild radius of the so-defined mass $M_{\mathrm{u}}$ of the universe.
\end{abstract}

\section{Introduction to a mass definition of the universe}

The question already often has been raised by cosmologists (see e.g. Tolman, 1918, Thirring, 1918, Jordan, 1947, Mashhoon, 1988, Mashhoon et al., 1984, Rosen \& Copperstock, 1992, Barbour, 1995) how the so-called mass $M_{\mathrm{u}}$ of an expanding universe, filled with homogeneously deposited matter subject to a Hubble flow, should semantically reasonably and physically adequately be conceived and what as such it may represent in form of a quantifiable cosmic number or a cosmic reality. How should this mass $M_{\mathrm{u}}$ be related to cosmological ingredients which are closer associated to the observable universe like the mean mass density, the cosmic scale variation with time or the Hubble parameter, or the so-called $\Omega$ - value, relating cosmic mass density and the critical density $\rho_{\text {crit }}$ ? Especially in an isotropically expanding, homogeneous universe with a vanishing or negative curvature parameter $k \leq 0$ in which the volume of the universe is infinite, the definition of such a quantity like $M_{\mathrm{u}}$ at first glance does not seem to be straightforward, perhaps not making sense at all.

Starting with suggestions made by Dirac (1937) and investigations carried out by Schuecking (1954) and Einstein \& Straus (1945) ideas were developing and concretizing that the masses of elementary particles as well as of stars might

^ Corresponding author: hfahr@astro.uni-bonn.de be somehow related to the large scale structure of the universe and its development in cosmic time. Much earlier already Mach (1883) had formulated as a physical requirement that the inertial mass of each particle just by its rigorous concept should somehow be related to the constellation of all other masses in the universe. Though Mach 's principle never up to the present completely entered into theoretical formulations of mass and gravity interacting with eachother, this principle already pointed to the probable fact that masses and their constellation in the universe should be related to eachother. This idea was later taken up by Thirring 1918 who was asking the question what the absolute inertial rest frame would be in the presence of rotating masses. In his view it should lead to identical geoidic deformations of the rotating earth regardless whether the earth rotates with respect to the universe with an angular frequency $\omega$, or whether the universe rotates with $-\omega$ with respect to the earth at rest.

To put these two cases on a physically conceivable basis, he compared two situations: a) the earth rotating with $\omega$ in the center of a universe at rest represented by a massive spherical shell with radius $R_{\mathrm{u}}$ and mass $M_{\mathrm{u}}$, and b) the earth at rest in the center of the universe represented identical to case a), however, this time rotating with $-\omega$ with respect to the earth. The result of this comparison, when extracted from the adequate Newtonian approximation of Einstein's field equations for rotating masses, Thirring (1918) (see also Mashhoon et al. 1984) found that at a space point close 
to the axis in the rotation plane the centrifugal or geodetic forces operating at the earth's equator in cases a) and b) are related by

$\mathbf{K}_{\mathrm{centri}}=X \cdot \mathbf{K}_{\mathrm{geod}}$

where the factor $X$ is calculated to be:

$X=\frac{4 G M_{\mathrm{u}}}{3 c^{2} R_{\mathrm{u}}}=\frac{2}{3} \frac{R_{\mathrm{s}, \mathrm{u}}}{R_{\mathrm{u}}}$,

with the associated Schwarzschild radius $R_{\mathrm{s}, \mathrm{u}}=2 G M_{\mathrm{u}} c^{-2}$ of the mass $M_{\mathrm{u}}$. This expresses the fact that the equivalence principle of rotational motions can only be satisfied in the present and upcoming world represented by values $M_{\mathrm{u}}$ and $R_{\mathrm{u}}$, if the value for $X$ would exactly evaluate to be constant and equal to: $X=1$. To preserve $X=1$ in an expanding universe would, however, require the expression $M_{\mathrm{u}} / R_{\mathrm{u}}$ to be a cosmological constant. This would mean that the total mass of the universe should scale with the increasing cosmic diameter $R_{\mathrm{u}}$, an unusual cosmological requirement.

In the following we shall not rely on the above indicated scaling of the mass of the universe with cosmic extent, we rather shall ask the more principle question, how mass $M_{\mathrm{u}}$ and extent $R_{\mathrm{u}}$ of an expanding universe should be properly defined. Hereby we simply rely on the cosmological principle according to which each space point in the universe is equivalent to any other cosmic space point concerning its being surrounded by matter-filled cosmic space, and shall ask by how much instantaneously countable matter, i.e. matter distributed on a 3-dimensional spacelike hypersphere of the universe, this selected point of the universe may in fact be surrounded. This specific question in our perspectives here is not connected with the problem of how long it may take, before the presence of distant masses in the universe can be communicated by electromagnetic or gravitational waves to our local standpoint in the universe, i.e. we do not intend to sum up retarded contributions of energy depositions in space. The question rather starts from the certainty and knowledge that any spacepoint in the universe is surrounded up to large cosmic distances by cosmic matter that is subject to a cosmological expansion dynamics. What then - may be called the instantaneous energy content of this spacepoint-related universe?

\section{Calculation of the proper radius}

The universe, taken as basis of our calculations, is assumed to be expanding isotropically and to have an isotropic curvature and a homogeneous energy distribution. As the total instantaneous energy of such a universe considered as an isolated system Tolman (1934) has given the following formula for the mass $M_{\mathrm{u}}$ of such a system:

$M_{\mathrm{u}}(t) c^{2}=\int^{3}\left[\rho(t) c^{2}+3 p(t)\right] \sqrt{-g_{3}} \mathrm{~d}^{3} V$,

where $\rho(t)$ and $p(t)$ denote mass density and pressure of the cosmic matter, and where $\mathrm{d}^{3} V_{0}=\sqrt{-g_{3}} \mathrm{~d}^{3} V$ denotes the differential of the spacelike 3-D proper volume.
In later phases of an evolving and expanding universe rather than pressure (n.b. matter becomes pressure-free!) as forms of positive energy representations, in addition to rest mass energy distributions of baryonic or dark matter type, also the kinetic or dynamic energy connected with the cosmological expansion would need to be considered. In an homologously expanding universe seen from any arbitrary spacepoint matter comoving with the cosmic expansion in its surroundings shows up with a Hubble flow. As judged from this spacepoint comoving matter at a distance $r$ thus also represents kinetic energy in form of its Hubble motion with a centripetal velocity $v_{\mathrm{c}}(r)=H(t) \cdot r$. Thus for a pointrelated energy balance account this form of Hubble-induced kinetic energy needs to be counted in addition to rest mass energy. To calculate the point-related total energy balance one may describe the surroundings of an arbitrary spacepoint, similar to the central spacepoint in the stellar interior, as surrounded by metrically relevant, space-curving energy. For such point-related balance the metric tensor elements of the so-called inner Schwarzschild metric tensor for the point-related cosmic matter distribution can be applied.

Therefore adding up the above mentioned energies in the whole universe filled with a pressure-less matter surrounding an arbitrary space point up to a distance $R_{\mathrm{u}}$ may then lead one instead of Equ. (3) to the following expression:

$M_{\mathrm{u}}\left(t, R_{\mathrm{u}}\right) c^{2}=\int^{3}\left[\gamma(r) \rho_{0}(t) c^{2}\right] \sqrt{-g_{3}} \mathrm{~d}^{3} V$,

where $\rho_{0}(t)$ denotes the homogeneous rest mass density which is variable only with cosmic time $t$, but not with space coordinates, where $\gamma(r)^{-2}=1-\left(v_{\mathrm{c}}(r) / c\right)^{2}$ is the Lorentz factor evaluated for the local expansion velocity $v_{\mathrm{c}}(r)=H(t) r$ with $H(t)=v_{\mathrm{c}}(r) / r=\left(1 / R_{\mathrm{u}}\right) \mathrm{d} R_{\mathrm{u}} / \mathrm{d} t$, and where $\mathrm{d}^{3} V_{0}=\sqrt{-g_{3}} \mathrm{~d}^{3} V$ is the local differential spacelike proper volume of space given through the determinant of the 3-D part of the inner Schwarzschild-metric tensor which yields the worldline element $\mathrm{d} s$ in the form (see e.g. Stephani 1988):

$$
\begin{aligned}
\mathrm{d} s^{2}=\exp (\lambda(r)) \mathrm{d} r^{2} & +r^{2}\left[\mathrm{~d} \vartheta^{2}+\sin ^{2} \vartheta \mathrm{d} \varphi^{2}\right]- \\
& -\exp (\nu(r)) c^{2} \mathrm{~d} t^{2}
\end{aligned}
$$

and the proper volume in the form:

$$
\begin{aligned}
\mathrm{d}^{3} V_{0}=\sqrt{-g_{\mathrm{rr}} g_{\vartheta \vartheta} g_{\varphi \varphi}} & \mathrm{d} r \mathrm{~d} \vartheta \mathrm{d} \varphi= \\
& =\sqrt{\exp \left(\lambda(r) r^{4} \sin ^{2} \vartheta\right.} \mathrm{d} r \mathrm{~d} \vartheta \mathrm{d} \varphi .
\end{aligned}
$$

The Robertson-Walker metric usually taken to describe the spacetime metrics in a homogeneous universe in this specific case of a space-related view has been replaced in view of the specific question posed here by the inner Schwarzschild metric which here is used up to the metric infinity with no need for a smooth connection to the RobertsonWalker metric. Then from Equ. (4) one obtains the total mass of the expanding universe with pressure-less matter and homogeneous density distribution in the form:

$M_{\mathrm{u}}(t) c^{2}=4 \pi \rho_{0}(t) c^{2} \int_{0}^{R_{\mathrm{u}}} \frac{\exp (\lambda(r) / 2) r^{2} \mathrm{~d} r}{\sqrt{1-\left(\frac{H r}{c}\right)^{2}}}$. 
Here the full energy density of cosmologically moving matter at $\mathrm{r}$ as seen from an arbitrary spacepoint at $r=0$ is given by $\epsilon=\gamma(r) \rho_{0} c^{2}$ where $\gamma(r)^{-2}=1-(H r / c)^{2}$ is the local Lorentz factor of the source point matter. The metric function $\exp [\lambda(r)]$ for the inner Schwarzschild metric of a pressure-less matter universe, using the analogy to the stellar case and following Stephani (1988), is given by:

$\exp [-\lambda(r)]=1-\frac{8 \pi G}{r c^{2}} \rho_{0} \int_{\mathrm{o}}^{r} \gamma(x) x^{2} \mathrm{~d} x$,

and thus allows to obtain the total mass of the universe in the following form:

$$
\begin{aligned}
& M_{\mathrm{u}}(t)=4 \pi \rho_{0}(t) \times \\
& \times \int_{0}^{R_{\mathrm{u}}} \frac{r^{2}}{\sqrt{1-\frac{8 \pi G}{r c^{2}} \rho_{0} \int_{\mathrm{o}}^{r} \gamma(x) x^{2} \mathrm{~d} x} \sqrt{1-\left(\frac{H r}{c}\right)^{2}}} \mathrm{~d} r .
\end{aligned}
$$

As one can see from the above expression, a singularity appears in the integrand at a sourcepoint distance where the line element $\mathrm{d} s_{\mathrm{r}}=\sqrt{g_{\mathrm{rr}}} \mathrm{d} r$ tends to infinity, i.e. at some outer critical radius $R_{\mathrm{u}}$ given by the following implicit relation:

$\frac{c^{2}}{8 \pi G \rho_{0}}=\frac{1}{R_{\mathrm{u}}} \int_{\mathrm{o}}^{R_{\mathrm{u}}} \frac{1}{\sqrt{1-\left(\frac{H x}{c}\right)^{2}}} x^{2} \mathrm{~d} x$,

and reminding that the above integral is solved by:

$$
\begin{aligned}
& \int_{\mathrm{o}}^{R_{\mathrm{u}}} \frac{1}{\sqrt{1-\left(\frac{H x}{c}\right)^{2}}} x^{2} \mathrm{~d} x=\left(\frac{c}{H}\right)^{3} \int_{0}^{\frac{H R_{\mathrm{u}}}{c}} \frac{\xi^{2} \mathrm{~d} \xi}{\sqrt{1-\xi^{2}}}= \\
& \left(\frac{c}{H}\right)^{3}\left(\frac{1}{2} \arcsin \frac{H R_{\mathrm{u}}}{c}-\frac{H R_{\mathrm{u}}}{2 c} \sqrt{1-\left(\frac{H R_{\mathrm{u}}}{c}\right)^{2}}\right.
\end{aligned}
$$

and going now to the horizon limit, where $c=H R_{\mathrm{u}}$ must be expected, would then bring us to:

$R_{\mathrm{u}}^{2} \frac{\pi}{4}=\frac{c^{2}}{8 \pi G \rho_{0}}$

or yielding:

$R_{\mathrm{u}}=\frac{1}{\pi} \sqrt{\frac{c^{2}}{2 G \rho_{0}}}$.

This result can also be interpreted as saying that a scaling of the density $\rho_{0}$ with the co-defined critical radius $R_{\mathrm{u}}$ of the universe holds according to the following relation:

$\rho_{0}\left(R_{\mathrm{u}}\right)=\frac{c^{2}}{2 \pi^{2} G R_{\mathrm{u}}^{2}}$.

Without the kinetic energy of the Hubble flow taken into account we instead of the above result would obtain from Equ. (10) the following simplified expression:

$\frac{c^{2}}{8 \pi G \rho_{0}}=\frac{1}{R_{\mathrm{u}}} \int_{\mathrm{o}}^{R_{\mathrm{u}}} x^{2} \mathrm{~d} x=\frac{1}{3} R_{\mathrm{u}}^{2}$,

leading to the result very similar to that obtained in Equ. (14), namely given by:

$\rho_{0}\left(R_{\mathrm{u}}\right)=\frac{3 c^{2}}{8 \pi G R_{\mathrm{u}}^{2}}=\frac{3 \pi}{4}\left(\frac{c^{2}}{2 \pi^{2} G R_{\mathrm{u}}^{2}}\right)$.

The difference between Hubble flow taken and not taken into account only amounts to a factor $(3 \pi / 4)$.

\section{Calculation of the proper mass}

First let us take up the results for the case that the Hubble kinetics is not taken into account and calculate the proper mass with Equ. (9) by:

$M_{\mathrm{u}}(t)=4 \pi \rho_{0}(t) \int_{0}^{R_{\mathrm{u}}} \frac{r^{2}}{\sqrt{1-\frac{8 \pi G}{r c^{2}} \rho_{0} \int_{\mathrm{o}}^{r} x^{2} \mathrm{~d} x}} \mathrm{~d} r$.

Using the result of Equ. (15) for $R_{\mathrm{u}}$, one then obtains:

$$
\begin{aligned}
M_{\mathrm{u}}(t)= & 4 \pi \rho_{0}(t) R_{\mathrm{u}}^{3} \int_{0}^{1} \frac{\xi^{2}}{\sqrt{1-\xi^{2}}} \mathrm{~d} \xi= \\
& 4 \pi \rho_{0}(t) R_{\mathrm{U}}^{3} \frac{\pi}{4},
\end{aligned}
$$

which with Equ. (16) leads to the result:

$M_{\mathrm{u}}(t)=\pi^{2} \rho_{0}(t) R_{\mathrm{u}}^{3}=\frac{3 \pi^{2} c^{2}}{8 \pi G} R_{\mathrm{u}}$,

which expresses the fact that the above defined mass of the universe scales with the critical radius of the universe $R_{\mathrm{u}}$ defined by Equ. (4).

Using now the above relation one finds that Thirring's relation given by Equ. (2) with the above introduced quantities appears to be fulfilled, since one obtains:

$X=\frac{4 G M_{\mathrm{u}}}{3 c^{2} R_{\mathrm{u}}}=\frac{4 G}{3 c^{2} R_{\mathrm{u}}} \frac{3 \pi^{2} c^{2}}{8 \pi G} R_{\mathrm{u}}=\frac{\pi}{2}$.

Now we shall take also into account the Hubble kinematics. Therefore using now, instead of the above, the radius of the universe given by Equ. (13) one may then also calculate for a universe with Hubble flow the total mass of the universe from Equ. (9) in the form:

$M_{\mathrm{u}}(t)=4 \pi \rho_{0}(t) \int_{0}^{R_{\mathrm{u}}} \frac{r^{2} \mathrm{~d} r}{\sqrt{1-f_{1}(r) f_{2}(r)} \sqrt{f_{3}(r)}}$,

with

$f_{1}(r)=\frac{4}{\pi r R_{\mathrm{u}}^{2}}\left(\frac{c}{H}\right)^{3}$,

$f_{2}(r)=\frac{1}{2} \arcsin \frac{H r}{c}-\frac{H r}{2 c} \sqrt{1-\left(\frac{H r}{c}\right)^{2}}$,

$f_{3}(r)=1-\left(\frac{H r}{c}\right)^{2}$,

which leads to the following expression:

$$
\begin{aligned}
& M_{\mathrm{u}}(t)=4 \pi \rho_{0}(t)\left(\frac{c}{H}\right)^{3} \times \\
& \times \int_{0}^{\xi_{\mathrm{u}}} \frac{\xi^{2} \mathrm{~d} \xi}{\sqrt{1-\frac{2}{\pi \xi \xi_{\mathrm{u}}^{2}}\left[\arcsin \xi-\xi \sqrt{1-\xi^{2}}\right]} \sqrt{1-\xi^{2}}}
\end{aligned}
$$

where the notations $\xi=H r / c$ and $\xi_{\mathrm{u}}=H R_{\mathrm{u}} / c$ have been used.

It turns out that the above formula for the mass of the universe as function of the world radius $R_{\mathrm{u}}$ in the limit $\xi_{\mathrm{u}} \rightarrow 1$ is numerically given by:

$\lim _{\xi_{\mathrm{u}} \rightarrow 1} M_{\mathrm{u}}(t)=4 \pi \rho_{0}(t) R_{\mathrm{u}}^{3} \cdot 1.6150446$, 
and using now the result from given in Equ. (14), i.e. $\rho_{0}\left(R_{\mathrm{u}}\right)$ $=c^{2} /\left(2 \pi^{2} G R_{\mathrm{u}}^{2}\right)$, then yields the astonishing result:

$M_{\mathrm{u}}(t)=1.6150446 \frac{4 \pi c^{2} R_{\mathrm{u}}^{3}}{2 \pi^{2} G R_{\mathrm{u}}^{2}} \approx \frac{c^{2}}{G} R_{\mathrm{u}}$,

revealing the fact that the above-defined mass of the universe $M_{\mathrm{u}}(t)$ in the form it is conceived again scales with the corresponding radius of the universe.

On the other hand, turning things around, this also states that the radius of the universe is about equal to the Schwarzschild radius of this mass $M_{\mathrm{u}}(t)$ of the universe, since one finds:

$R_{\mathrm{u}}=\frac{1}{2} \frac{2 G M_{\mathrm{u}}(t)}{c^{2}} \approx 0.5 \cdot R_{\mathrm{s}, \mathrm{u}}$.

\section{Conclusions}

We have shown that with the use of an analogy to the inner stellar Schwarzschild metric applied to the cosmic matter distribution one can arrive at a reasonable definition of what could be called the mass $M_{\mathrm{u}}(t)$ of the universe. Interestingly enough, this mass $M_{\mathrm{u}}(t)$ scales with the critical radius $R_{\mathrm{u}}$ of the universe introduced by Equ.(10) which was a request already since the works by Mach (1983), Thirring (1918) (see also Mashhoon et al. 1984, Barbour \& Pfister 1995, Wesson 1999, Hoyle 1990, 1992, Jammer 2000 or Fahr \& Heyl 2006). Furthermore the finding that the average density $\rho_{0}$ of the universe turns out to be scaling with the reciprocal of the square of the above defined critical radius $R_{\mathrm{u}}$ of the universe just fulfills the request for an economical universe with vanishing total energy as was discussed by Overduin \& Fahr (2003), Fahr (2004) and Fahr \& Heyl (2006). In the near future we have plans to also consistently include into the considerations the cosmic vacuum energy density to see what changes might result from that for the above derived concepts.

An outstanding problem perhaps may still be to better understand the natural philosophical semantics and the logical or physical implications of the above given definitions of $M_{\mathrm{u}}$ and $R_{\mathrm{u}}$. One should perhaps notice that in the above derived formulae no strict physical reason is presented why cosmic matter density $\rho_{0}$ scales with $R_{\mathrm{u}}^{-2}$. We only have derived above a critical world radius $R_{\mathrm{u}}=R_{\mathrm{u}}\left(\rho_{0}\right)$ which is given as function of the prevailing cosmic matter density $\rho_{0}$ in a way such that the relation $\rho_{0} \sim R_{\mathrm{u}}^{-2}$ is fulfilled. Only when by some cosmological evolution process not discussed here this density changes by its value in time, making $\rho_{0}=\rho_{0}(t)$, then it turns out that the accordingly changing critical radius $R_{\mathrm{u}}=R_{\mathrm{u}}\left(\rho_{0}(t)\right)$ also changes, as if the relation $\rho_{0} \sim R_{\mathrm{u}}^{-2}$ would be valid. The many cosmological implications of that shall have to be discussed in more detail in forthcoming considerations.

\section{References}

Barbour ,J.B.: 1995, in: J.B. Barbour, H. Pfister, General relativity as a perfectly Machian theory, p. 214
Barbour, J., Pfister, H.: 1995, in: 'Mach' principle: from Newton's bucket to quantum gravity', Birkhäuser Verlag, Berlin

Dirac, P.A.M.: 1937, Nature 139, 323

Einstein, A., Straus, E.G.: 1945, Rev.Modern Phys. 17, 120

Fahr, H.J.: 2004, in: W.Loeffler, P.Weingartner (eds.), Knowledge and Belief - Wissen und Glauben, 26.th Int.Wittgenstein Symposium, öbv\&hpt, Wien

Fahr, H.J., Heyl, M.: 2006, Astrophys.Space Sci., submitted

Hoyle, F.: 1990, Astrophys. Space Sci. 168, 59

Hoyle, F.: 1992, Astrophys. Space Sci. 198, 195

Jammer, M.: 2000, in: Concepts of Mass in contemporary Physics and Philosophy, Princeton University Press, Princeton

Mach, E.: 1983, in: Die Mechanik in ihrer Entwicklung, historischkritisch dargestellt, F.A.Brockhaus, Leipzig

Mashhoon, B., Hehl, F.H., Theiss, D.S.: 1984, Gen.Rel.Grav. 16, 712

Overduin, J., Fahr, H.J.: 2003, Foundations of Physics Letters 16(2), 119

Rosen, N., Copperstock, F.I.: 1992, in: The mass of a body in general relativity, Class. Quantum Gravity, 9, p.2657

Schuecking, E.: 1954, Zeitschrift f. Physik 137, 595

Stephani, H.: 1988, in: Allgemeine Relativitätstheorie, VEB Deutscher Verlag der Wissenschaften, Berlin, p.113

Thirring, H.: 1918, Phys. Zeitschrift 19, 33

Tolman, R.: 1934, in: Relativity, Thermodynamics and Cosmology, Clarendon Press, Oxford, p.235

Wesson, P.S.: 2000, Observatory 120, 59 